\title{
The combination of clofarabine, etoposide, and cyclophosphamide shows limited efficacy as a bridge to transplant for children with refractory acute leukemia: Results of a monitored prospective study
}

\author{
Jacek Toporski ${ }^{1}$, Ladislav Król ${ }^{1}$, Josefina Dykes ${ }^{2}$, Yvonne Håkansson ${ }^{1}$, Cornelis Pronk ${ }^{1}$, \\ and Dominik Turkiewicz ${ }^{1}$ \\ ${ }^{1}$ Skåne University Hospital Lund \\ ${ }^{2}$ Skåne University Hospital Labmedicin Skane
}

July 16, 2020

\begin{abstract}
Background Clofarabine has been shown to effectively induce remission in children with refractory leukemia. We conducted a prospective study to explore the use of clofarabine-based chemotherapy as a bridge-to-transplant approach. Methods Children with refractory acute leukemia were enrolled to receive two induction courses of clofarabine, etoposide, and cyclophosphamide (CloEC). Responding patients were scheduled for T-cell depleted haploidentical hematopoietic stem cell transplantation (HSCT). The primary objective was to improve survival by achieving sufficient disease control to enable stem cell transplantation. Secondary objectives were to evaluate safety and toxicity. Results Seven children with active disease entered the study. Two children responded to induction courses and underwent transplantation. Five children did not respond to induction: one died in progression after the first course; two received off-protocol chemotherapy and were transplanted; and two succumbed to progressive leukemia. All transplanted children engrafted and no acute skin graft-versus-host disease $>$ grade I was observed. One child is alive and well 7.5 years after the first CloEC course. One child developed fulminant adenovirus hepatitis and died in continuous complete remission 7 months after start of induction. Two children relapsed and died 6.5 and 7.5 months after enrollment. Infection was the most common toxicity. Conclusions CloEC can induce responses in some patients with refractory acute leukemia but is highly immunosuppressive, resulting in substantial risk of life-threatening infections. In our study, haploidentical HSCT was feasible with sustained engraftment. No clinically significant organ toxicity was observed. Also, repeating CloEC probably does not increase the chance of achieving remission.
\end{abstract}

\section{INTRODUCTION}

Despite improvement in survival of children with acute leukemia (AL), a significant number of such patients still experience primary or secondary resistance to treatment. ${ }^{1,2}$ Children failing to achieve remission and those relapsing after previous allogeneic hematopoietic stem cell transplantation have a short life expectancy, and palliative treatment remains the only reasonable option, because in many cases escalation of conventional chemotherapy is no longer effective. Thus far, cell/immune therapies are applied primarily in B precursor acute lymphoblastic leukemia (ALL), whereas refractory T-cell acute lymphoblastic leukemia (T-ALL) and acute myeloblastic leukemia (AML) still represent an unmet challenge. ${ }^{3}$ Allogeneic hematopoietic stem cell transplantation (HSCT) seems to improve survival in children with high-risk acute leukemia. Better survival can in part be mediated by the graft-versus-leukemia (GvL) effect together with the anti-leukemic effect of the conditioning regimen. However, the efficacy of allogeneic HSCT is inversely correlated to the tumor burden. In patients without at least morphological remission at the time of transplantation, the risk of subsequent relapse is very high. Clofarabine has been shown to potently induce remissions in refractory cases of pediatric acute leukemia, both when given as a single drug and in combination with etoposide and 
cyclophosphamide. In the Nordic countries, clofarabine has not been incorporated in the primary treatment of acute leukemia in children, thus leukemic cells from patients who are refractory to multiple multidrug regimes have usually not been exposed to clofarabine. Still, responses induced by clofarabine-containing drug combinations are often transient in character. Nevertheless, even a temporary decrease in tumor load before an allogeneic HSCT could theoretically increase the potency of the GvL effect in reducing the risk of relapse, making this treatment modality a potential consolidation of clofarabine-induced responses. The short duration of clofarabine-elicited responses requires precise timing of the allogeneic HSCT. Transplantation with haploidentical donors offers such precision and may also lead to an enhanced GvL effect due to the HLA disparity between the donor and recipient. ${ }^{4-6}$ Our aim was to evaluate the safety and toxicity of intensified clofarabine-based multidrug remission induction for heavily pretreated children with $\mathrm{r} / \mathrm{r} \mathrm{AL}$, and also determine the potential of this approach to bridge these patients to a promptly timed haploidentical HSCT.

\section{PATIENTS AND METHODS}

\section{Target population}

The target population for this prospective study comprised children and adolescents referred to our institution with relapsed/refractory (r/r) AL (ALL or AML including sAML), who were aged > 1 and [?] 21 years at the time of enrollment. For both ALL and AML, refractoriness was defined as chemoresistant isolated or combined bone marrow relapse, relapse $>6$ months after allogeneic HSCT, or primary induction failure. Patients relapsing after allogeneic HSCT could be recruited directly. Patients relapsing after conventional treatment were to have at least one failed remission induction attempt before enrollment. Disease status was mandatory for inclusion and was assessed by both morphological examination and immunophenotyping prior enrollment. The following additional eligibility criteria were necessary for enrollment: cardiac output SF [?] 25\%; adequate renal function indicated by calculated creatinine clearance [?] $90 \mathrm{ml} / \mathrm{min} / 1.73 \mathrm{~m}^{2}$ (calculated by the Schwartz formula for estimated glomerular filtration rate [GFR]); adequate liver function indicated by serum bilirubin [?] $1.5 \times$ upper limit of normal (ULN); aspartate transaminase/alanine transaminase; alkaline phosphatase [?] 2.5x ULN; Lansky or Karnofsky performance status of [?] 70\%; a suitable adult haploidentical family member available for stem cell donation and fulfilling institutional criteria for blood and marrow donation. Informed consent and assent (adjusted to different age categories) from patients/parents were required for enrollment. The study was approved by the Swedish Ethical Review Authority (File no. 2009/83) and registered at clinicval.trials.gov (NCT01025778) and the European Union Drug Regulating Authorities Clinical Trials Database (EudraCT number 2009-012437-30), and it was conducted in accordance with the principles of the Helsinki Declaration.

\section{Treatment plan}

Re-induction CloEC chemotherapy consisted of clofarabine $40 \mathrm{mg} / \mathrm{m}^{2} /$ day, etoposide $100 \mathrm{mg} / \mathrm{m}^{2} /$ day, and cyclophosphamide $340 \mathrm{mg} / \mathrm{m}^{2} /$ day, and it was given on five consecutive days. Children responding to the first course of CloEC received the second course and proceeded to transplantation. Children not responding to the first course were given the second course, and if that did result in fulfillment of the transplant criteria, an additional (third) course of CloEC was administered pending transplantation. Children not responding to the second course entered the palliative care program. Those with $>5 \%$ blasts on day 21 after the beginning of the first course were immediately started on the second course. Those with [?] $5 \%$ blasts in hypoplastic bone marrow $(\mathrm{BM})$ were re-evaluated once a week until regeneration occurred or the blasts increased, and then proceeded to the second course. The minimum interval between CloEC courses was 21 days.

The conditioning regimen consisted of clofarabine $200 \mathrm{mg} / \mathrm{m}^{2}$ or fludarabine $150 \mathrm{mg} / \mathrm{m}^{2}$, thiotepa $10 \mathrm{mg} / \mathrm{kg}$, and melphalan $120 \mathrm{mg} / \mathrm{m}^{2}$, and it was given between days -8 and -1 prior to grafting. Serotherapy with ATG (Fresenius) at a total dose of $30 \mathrm{mg} / \mathrm{kg}$ was administered from day -12 to day -10 before grafting. For patients with ALL, the preferred donor was always the mother if there was no medical contraindication for donation. For AML patients, donor selection was based on KIR mismatch: if mismatch was observed, the mother was the first choice and the father second choice; if no mismatch was present, the mother was 
chosen. The donors were screened, evaluated, and accepted by an independent team of adult hematologists.

Peripheral blood stem cells were mobilized with G-CSF, and all donors were harvested twice. The collected cells were processed with the CliniMACS system for immunomagnetic cell separation (Miltenyi Biotec, Gladbach, Germany). The first harvest entailed selection of CD34+ cells, whereas the second harvest was for depletion of $\mathrm{T}$ cells expressing the $\alpha \beta$ chains of $\mathrm{T}$ cell receptor (TCR). Maximum allowed dose of TCR $\alpha \beta+$ cells was set at $5 \times 10^{5}$ cells $/ \mathrm{kg}$. A single dose $\left(375 \mathrm{mg} / \mathrm{m}^{2}\right)$ of anti-CD20 antibody (Rituximab) was administered on day +1 after grafting instead of performing B-cell depletion in vivo. Graft-versushost prophylaxis included the above-mentioned serotherapy, graft processing, and a short course of MMF $600 \mathrm{mg} / \mathrm{m}^{2}$ tid given from day -1 to day +28 if the number of residual $\alpha \beta$ TCR positive cells in the graft exceeded $2.5 \times 10^{4} / \mathrm{kg}$ of donor's body weight.

\section{Definition of response}

Response to each course of CloEC was evaluated to determine whether to continue the treatment or proceed to transplant. The response criteria for ALL patients indicated morphological remission: $<5 \%$ of blasts in BM aspiration and signs of BM regeneration. For AML patients, the criteria were no blasts in PB and $<25 \%$ blasts in BM aspiration.

\section{Supportive care}

Before and after transplantation, all children receivedPneumocystis pneumonia and antifungal prophylaxis according to institutional guidelines. Irradiated blood products were used for transfusion support. Together with the CloEC, all patients received dexamethasone, both as antiemetic prophylaxis and as prophylaxis for cytokine release syndrome. No antibacterial prophylaxis was given, although an aggressive empirical treatment of febrile neutropenia episodes was mandatory. After transplantation, the patients were screened at least weekly for CMV, AdV, and EBV. If viral DNA was detected, the number virus copies was monitored, and appropriate antiviral treatment was started when indicated. All patients with IgG specific for varicella-zoster or herpes simplex viruses received prophylactic acyclovir according to institutional guidelines. Defibrotide was not used as VOD prophylaxis. Human granulocyte colony-stimulating factor was not used to enhance neutrophil recovery after transplantation.

\section{RESULTS}

\section{Study cohort}

The study included seven patients with refractory acute leukemia: two males and five females aged 3 to 16 years. Five of the patients were diagnosed with ALL (four BCP-ALL and one T-ALL ) and two with AML (one de novo and one sAML after previous treatment of BCP-ALL). All patients had active disease, i.e., $>5 \%$ of leukemic blasts in bone marrow at the time of enrollment. One patient had primary refractory disease and never achieved complete remission before the first course of CloEC. One patient had a very early relapse of T-ALL (while on treatment) and was recruited directly to the study. The remaining patients had either first or subsequent refractory relapse and had failed two or more lines of treatment, in two patients including allogeneic HSCT. Characteristics of the patients are presented in Table 1.

\section{Response to CloEC}

All children received the first induction chemotherapy according to the protocol. No deaths related to the clofarabine-containing induction were observed. Two of the seven patients (both with BCP- ALL) responded to the first course of CloEC: one proved to be negative for $\mathrm{MRD}$, and the other was in morphological remission and highly positive for MRD. The MRD-negative patient continued the treatment according to the protocol and was transplanted after the second CloEC. As decided by the treating physician, the highly MRD-positive patient received treatment that deviated from the protocol, proceeding directly to transplantation after the first course of CloEC.

The remaining five children did not respond to the first course of CloEC. One of those non-responders was diagnosed with possible cerebro-pulmonary invasive fungal infection and was found to have leukemic blasts in 
peripheral blood. The parents declined further treatment, and the patient died of progressive disease 1 month after the first CloEC. In one patient with sAML who was recruited to the study in relapse after previous haploidentical transplantation, there was no measurable effect of the first course of CloEC on the tumor burden; the treating physician considered it unreasonable to continue with the second course stipulated in the protocol. This patient instead received liposomal daunorubicin, fludarabine, and cytarabine (FLADx), and achieved MRD-negative remission and could finally proceed to transplant.

Three of the five children who did not achieve remission after the first course of CloEC received the second course, but none of them responded. Two of those three continued with only palliative treatment after the second CloEC and died of progressive disease 57 and 132 days after the first course. One child was in a very good clinical condition after the second CloEC, and disease evaluation at this time point showed a reduction in blasts from $80 \%$ to below $30 \%$; as decided by the treating physician, despite not fulfilling response criteria after the second course of CloEC, this patient received the third course but without further decrease in percentage of blasts. Inasmuch as the clinical condition of this patient was still very good, the fourth course of chemotherapy (FLADx) was given, after which further reduction in blast percentage was observed. Despite still not being in morphological remission after the fourth course of chemotherapy, the patient proceeded to transplant.

In all, two of the seven enrolled children achieved protocol-defined response criteria. One additional patient (sAML) who went into remission after CloEC was changed to FLADx. Re-induction with CloEC and the patients' responses are summarized in Table 2.

\section{Transplant outcome}

Four children underwent haploidentical HSCT 43, 76, 78, and 151 days, respectively, after start of the first course of CloEC. Three of them fulfilled proceed-to-transplant criteria, and one was transplanted according to the treating physician's decision despite not being in morphological remission. All donors were mothers. The numbers of transplanted CD34+ cells ranged from 4.45 to $10.6 \times 10^{6} / \mathrm{kg}$ and residual $\alpha \beta$ TCR+ lymphocytes from 5.0 to $102 \times 10^{3} / \mathrm{kg}$. All children showed sustained engraftment. One patient is alive and well 7.5 years after transplantation. Two children relapsed on days +55 and +128 post-transplant and ultimately died of progressive leukemia. One child developed AdV-induced fulminant hepatitis and died of liver failure in complete remission. Details of transplantation and post-transplant follow-up are presented in Table 3.

\section{Toxicity - CloEC courses}

The most common toxicity was profound bone marrow aplasia (grade 4 neutropenia and thrombocytopenia), observed in all patients. Microbiologically verified bacterial infections were registered in three of the seven patients after the first course of $\mathrm{CloEC}$ and in two out of four after the second course. One patient was diagnosed with a possible cerebro-pulmonary invasive fungal infection after the first course. Viral infections were observed in two patients after the second course (one CMV reactivation and one AdV infection). Liver toxicities were limited to transient elevation of transaminases classified as grade 4 in only one patient. No cases of VOD or hyperbilirubinemia were recorded. Only one case of mild mucositis was observed, and only one patient after each course required parenteral nutrition.

\section{Toxicity - transplantation}

Septicemia was observed in two of the four transplanted patients, in two patients with gram-positive pathogens, and in one patient with gram-negative pathogens. One patient had been diagnosed with biopsyverified invasive cerebro-pulmonary mucormycosis. Viral infections were observed in all patients; two of them had infections with multiple viral pathogens, but no EBV reactivation was detected. All four transplanted patients experienced a period of profound pancytopenia. Liver toxicities noted as elevations in transaminases and/or bilirubin were observed in three of the patients, although none of them developed VOD. Grade 3 to 4 mucositis was recorded in two patients, nutritional support was required in three, one patient developed grade II hemorrhagic cystitis, and one showed sagittal sinus thrombosis probably caused by invasive fungal infection in the CNS. Acute grade I skin GvHD was diagnosed in only one patient. No chronic GvHD was 
observed. Toxicities are summarized in Table 4.

\section{DISCUSSION}

Current treatment protocols for both ALL and AML in children are effective, and the majority of children can be saved by intensive conventional chemotherapy with/or without stem cell transplantation. ${ }^{1,2}$ Indeed, the newest approaches, such as use of bispecific antibodies or cell therapy, are successful even for therapy-resistant ALL. ${ }^{5}$ It is still an open question whether immunotherapy alone can suffice to achieve long-lasting remission particularly in refractory disease. ${ }^{7}$ A number of immunotherapies for $\mathrm{r} / \mathrm{r}$ AML are under investigation, but the best immunotherapy for this condition that is currently available entails attempting to control the disease and subsequently performing stem cell transplantation. ${ }^{8}$ Thus stem cell transplantation seems to be the most widely applied and proven curative procedure for children with refractory AL. ${ }^{9-12}$ It is commonly accepted that the better the disease control before HSCT, ${ }^{13-16}$ the better the expected outcome. Thus in $\mathrm{r} / \mathrm{r} \mathrm{AL}$, the main focus is on effective reduction of blast cell load that can potentially bridge the patients to a salvage procedure: HSCT alone or cell therapy with or without subsequent HSCT. Immunotherapy was not available when we designed and performed our study, and the only possible approach to bridge the patients to HSCT at that time was intensified re-induction chemotherapy. ${ }^{17,18}$ Clofarabine, both alone or in combination with other agents, is known to be effective and is recommended for relapsing AL, with acceptable toxicity. The salvage therapy is nearly always given to heavily pretreated children, including those with previous HSCT, and such patients often have pre-existing organ toxicities. We chose the combination of clofarabine, etoposide, and cyclophosphamide based on promising published data showing an acceptable toxicity profile (even in HSCT) ${ }^{6}$ However, our results suggest that the applicability of the protocol for this demanding patient group was limited by the treating physicians' pursuit of an individualized approach, and a lack of confidence in repeating CloEC if the first course did not result in a substantial response. Two of the seven patients deviated significantly from the CloEC protocol: one of them was transplanted already after the first course, and the other one received a different second course. One patient received the third CloEC off protocol (despite not fulfilling the required response criteria) and proceeded to transplantation while not in morphological remission; this decision reflected the patient's good clinical condition despite a high treatment burden, and the treating physician's hope that the GvL effect after haploidentical HSCT might overcome a high tumor load at transplantation. Considering the two CloEC responders (both with BCP-ALL), one was transplanted already after the first course of CloEC despite still having a high MRD $(>1 \%)$ before transplantation. The other non-responder was in complete morphological remission but had $1 \times 10^{-4}$ positive MRD after the first course and undetectable MRD after the second course; this is the only long-term survivor in our cohort. The children with AML and T-ALL did not respond and progressed, although one of them could be salvaged by changing chemotherapy from CloEC to FLADx; this patient was finally transplanted in morphological remission but died in remission of AdV hepatitis. Our results suggest that CloEC should probably not be repeated in patients showing no response after the first course, whereas repeating CloEC may further decrease the pre-transplant tumor load in responding patients.

No deaths related to CloEC toxicity were observed, and, in all children who died before being transplanted, death was due to progressive disease. However, the toxicity of the courses of CloEC was significant and mainly related to the profound immuno- and myelosuppression. Still, it is plausible that, together, both CloEC and previous treatments (including allogeneic HSCT in two patients) contributed to the immunosuppressive state of the patients. Contrary to some previous observations, ${ }^{6,17}$ we found no significant liver toxicity, neither during induction phase nor after transplantation. In all cases, the timing of the transplantation could be adjusted to the changing situations of the children. There was no unnecessary delay in transplantation due to logistical difficulties, reflecting the flexibility of this transplant modality. Ex-vivo T-cell depletion effectively prevented clinically relevant GvHD, even though the pharmacological prophylaxis was limited, MMF based, and given only during the first 4 weeks after transplantation.

In conclusion, the combination of clofarabine, etoposide, and cyclophosphamide did not seem to be a universal treatment for $\mathrm{r} / \mathrm{r}$ AL: the only children responding to CloEC in our study were those diagnosed with BCPALL. Nevertheless, this particular group of patients may be offered salvage immunotherapy with either 
bi-specific T-cell engaging CD19 antibodies or chimeric antigen receptor (CAR) T-cells, an approach that seems more reasonable than exposing such patients to CloEC. Haploidentical HSCT remains a viable option in cases in which flexibility in planning the transplantation essential, although further studies are needed to assess the anti-leukemic potential of such HSCT.

\section{CONFLICT OF INTEREST}

The authors have no conflict of interest.

\section{ACKNOWLEDGMENTS}

Sanofi Genzyme partly supported this investigator-sponsored trial by providing clofarabine (GZ-2010-10157). Representatives of Sanofi Genzyme were involved in writing the study protocol but were not involved in selection of the patients or collection, management, and analysis of the data.

\section{REFERENCES}

1. Oskarsson T, Soderhall S, Arvidson J, et al. Relapsed childhood acute lymphoblastic leukemia in the Nordic countries: prognostic factors, treatment and outcome. Haematologica. 2016;101(1):68-76.

2. Tallen G, Ratei R, Mann G, et al. Long-term outcome in children with relapsed acute lymphoblastic leukemia after time-point and site-of-relapse stratification and intensified short-course multidrug chemotherapy: results of trial ALL-REZ BFM 90. J Clin Oncol.2010;28(14):2339-2347.

3. Gore L, Locatelli F, Zugmaier G, et al. Survival after blinatumomab treatment in pediatric patients with relapsed/refractory B-cell precursor acute lymphoblastic leukemia. Blood Cancer J.2018;8(9):80.

4. LaBelle JL, Cunningham JM. Stem cell transplant as an immunomodulatory tool for children with hematologic malignancies.Am Soc Clin Oncol Educ Book.2013:10.1200/EdBook_AM.2013.1233.e1347.

5. Wyatt KD, Bram RJ. Immunotherapy in pediatric B-cell acute lymphoblastic leukemia. Hum Immunol. 2019;80(6):400-408.

6. Locatelli F, Testi AM, Bernardo ME, et al. Clofarabine, cyclophosphamide and etoposide as single-course re-induction therapy for children with refractory/multiple relapsed acute lymphoblastic leukaemia. $\mathrm{Br} J$ Haematol. 2009;147(3):371-378.

7. Taraseviciute A, Broglie L, Phelan R, Bhatt NS, Becktell K, Burke MJ. What is the Role of Hematopoietic Cell Transplantation (HCT) for Pediatric Acute Lymphoblastic Leukemia (ALL) in the Age of Chimeric Antigen Receptor T-Cell (CART) Therapy? Journal of pediatric hematology/oncology. 2019;41(5):337-344.

8. Przespolewski A, Szeles A, Wang ES. Advances in immunotherapy for acute myeloid leukemia. Future Oncol. 2018;14(10):963-978.

9. Bertaina A, Zecca M, Buldini B, et al. Unrelated donor vs HLA-haploidentical $\alpha / \beta$ T-cell- and B-celldepleted HSCT in children with acute leukemia. Blood. 2018;132(24):2594-2607.

10. Erbey F, Akcay A, Atay D, Ovali E, Ozturk G. Comparison of outcomes after HLA-matched unrelated and alphabeta T-cell-depleted haploidentical hematopoietic stem cell transplantation for children with highrisk acute leukemia. Pediatr Transplant. 2018;22(4):e13192.

11. Sharma A, Kang G, Sunkara A, et al. Haploidentical Donor Transplantation Using a Novel Clofarabinecontaining Conditioning Regimen for Very High-risk Hematologic Malignant Neoplasms. J Pediatr Hematol Oncol. 2018;40(8):e479-e485.

12. Shelikhova L, Ilushina M, Shekhovtsova Z, et al. alphabeta T Cell-Depleted Haploidentical Hematopoietic Stem Cell Transplantation without Antithymocyte Globulin in Children with Chemorefractory Acute Myelogenous Leukemia. Biol Blood Marrow Transplant.2019;25(5):e179-e182. 
13. Eckert C, Henze G, Seeger K, et al. Use of Allogeneic Hematopoietic Stem-Cell Transplantation Based on Minimal Residual Disease Response Improves Outcomes for Children With Relapsed Acute Lymphoblastic Leukemia in the Intermediate-Risk Group. Journal of Clinical Oncology. 2013;31(21):2736-2742.

14. Berry DA, Zhou S, Higley H, et al. Association of Minimal Residual Disease With Clinical Outcome in Pediatric and Adult Acute Lymphoblastic Leukemia: A Meta-analysis. JAMA Oncol. 2017;3(7):e170580.

15. Araki D, Wood BL, Othus M, et al. Allogeneic Hematopoietic Cell Transplantation for Acute Myeloid Leukemia: Time to Move Toward a Minimal Residual Disease-Based Definition of Complete Remission? $J$ Clin Oncol. 2016;34(4):329-336.

16. Pulsipher MA, Langholz B, Wall DA, et al. Risk factors and timing of relapse after allogeneic transplantation in pediatric ALL: for whom and when should interventions be tested? Bone Marrow Transplant.2015;50(9):1173-1179.

17. Jeha S, Razzouk B, Rytting M, et al. Phase II study of clofarabine in pediatric patients with refractory or relapsed acute myeloid leukemia. J Clin Oncol. 2009;27(26):4392-4397.

18. Jeha S, Gaynon PS, Razzouk BI, et al. Phase II study of clofarabine in pediatric patients with refractory or relapsed acute lymphoblastic leukemia. J Clin Oncol. 2006;24(12):1917-1923.

\section{Hosted file}

TABLE 1 Toporski.docx available at https://authorea.com/users/342951/articles/469709-thecombination-of-clofarabine-etoposide-and-cyclophosphamide-shows-limited-efficacy-as-abridge-to-transplant-for-children-with-refractory-acute-leukemia-results-of-a-monitoredprospective-study

\section{Hosted file}

TABLE 2 Toporski.docx available at https://authorea.com/users/342951/articles/469709-thecombination-of-clofarabine-etoposide-and-cyclophosphamide-shows-limited-efficacy-as-abridge-to-transplant-for-children-with-refractory-acute-leukemia-results-of-a-monitoredprospective-study

\section{Hosted file}

TABLE 3 Toporski.docx available at https://authorea.com/users/342951/articles/469709-thecombination-of-clofarabine-etoposide-and-cyclophosphamide-shows-limited-efficacy-as-abridge-to-transplant-for-children-with-refractory-acute-leukemia-results-of-a-monitoredprospective-study

\section{Hosted file}

TABLE 4 Toporski.docx available at https://authorea.com/users/342951/articles/469709-thecombination-of-clofarabine-etoposide-and-cyclophosphamide-shows-limited-efficacy-as-abridge-to-transplant-for-children-with-refractory-acute-leukemia-results-of-a-monitoredprospective-study 